\title{
Capacitação de agentes comunitários de saúde para a detecção da demência na fase leve
}

\author{
Capacitating community health workers to detect dementia at the mild stage \\ Capacitación de agentes comunitarios de salud para detección de las demencias en la fase leve
}

\author{
Mariana de Castro Barbosa'; Emanuela Bezerra Torres Mattos"'; Rosilda Mendes ${ }^{\prime \prime \prime}$
}

\begin{abstract}
RESUMO
Objetivo: avaliar o processo de educação permanente na capacitação de agentes comunitários de saúde (ACS) para a deteç̧ão de pessoas com demência na fase leve. Método: descritivo, comparando o desempenho dos sujeitos antes e após a oficina de aprendizagem. Após a aprovação pelo Comitê de Ética em Pesquisa, foi realizada oficina de capacitação com oito ACS de uma unidade básica de saúde, da cidade de Santos, em 2018. Antes e após a oficina foram aplicados questionários semiestruturados. Os dados foram tratados a partir da análise temática. Resultados: a falta de conhecimento sobre a demência aponta para a necessidade de investimento em educação permanente dos ACS como uma importante ferramenta a ser utilizada na detecção de casos iniciais. Conclusão: a educação permanente pode contribuir para o maior número de casos identificados nas fases iniciais da demência na atenção básica de forma que os encaminhamentos necessários e tratamentos possíveis sejam iniciados precocemente de forma a beneficiar pessoas e/ou suas famílias.
\end{abstract}

Descritores: Agente comunitário de saúde; demência; educação permanente; política de saúde.

\begin{abstract}
Objective: to evaluate the continuing professional development (CPD) process in capacitation of community health workers (CHWs) to detect early dementias. Method: this descriptive study compared the performance of eight CHWs from a Basic Health Unit in Santos, by applying semi-structured questionnaires before and after a CPD workshop held in 2018, after approval by the research ethics committee. The data were processed using thematic analysis. Results: the CHWs' lack of knowledge about dementia points to the need to invest in continuing professional development as an important tool to be used to detect early cases. Conclusion: continued professional development can contribute to identification of more cases in the early stages of dementia in primary care, so that necessary referrals and possible treatment are initiated early to benefit individuals and/or their families.
\end{abstract}

Descriptors: Community health agent; dementia; permanent education; health policy.

\section{RESUMEN}

Objetivo: evaluar el proceso de educación permanente en la capacitación de agentes comunitarios de salud (ACS) para detección de personas con demencia en la fase leve. Método: estudio descriptivo, comparado el desempeño de las personas antes y después del taller de aprendizaje. Tras la aprobación del Comité de Ética en Investigación, se realizó un taller de capacitación con ocho ACS de una Unidad Básica de Salud de la ciudad de Santos, en 2018. Antes y después del taller, se aplicaron cuestionarios semiestructurados. Los datos fueron procesados desde el análisis temático. Resultados: la falta de conocimiento sobre la demencia apunta hacia la necesidad de inversión en educación permanente, por parte de los ACS, como una importante herramienta a ser utilizada en la detección de casos iniciales. Conclusión: la educación permanente puede contribuir a la identificación en la atención básica de mayor número de casos en las fases iniciales de la demencia de forma a que las derivaciones aplicables y los posibles tratamientos se inicien precozmente para poder beneficiar a las personas $y / o$ a sus familias.

Descriptores: Agente comunitario de salud; demencia; educación permanente; política de salud.

\section{INTRODUÇÃO}

Com a transição demográfica, a América Latina atingirá em 2025 um número aproximado de 57 milhões de indivíduos com mais de 60 anos $^{1}$. Estimativas apontam que, em 2010, havia 35,6 milhões de pessoas com algum subtipo de demência em todo o mundo. Cerca de 7,7 milhões de novos casos surgem a cada ano, o que implica um novo caso a cada quatro segundos, em algum lugar do mundo. As projeções de prevalência e incidência indicam que esse número continuará a crescer, particularmente entre os idosos mais velhos, assim como, também, nos países em transição demográfica, como é o caso do Brasil².

Na cidade de Santos, dados publicados pela prefeitura apontam a existência de aproximadamente 85 mil idosos no município, representando cerca de $20 \%$ da população total ${ }^{3}$.

'Terapeuta Ocupacional. Graduada, Universidade Federal de São Paulo. São Paulo, Brasil. E-mail: maricastro.mc@gmail.com

"Terapeuta Ocupacional. Doutora. Professora Adjunta, Universidade Federal de São Paulo. São Paulo, Brasil. E-mail: emanuelabtm@gmail.com

I"Biológa. Pós doutora. Professora Associada, Universidade Federal de São Paulo. Brasil. E-mail: rosildamendes@terra.com.br

IVAgradecimentos ao Conselho Nacional de Pesquisa e Desenvolvimento Tecnológico pela bolsa de Iniciação Cientifica que culminou com o presente artigo. 
Dessa forma, é primordial a inserção da educação permanente em saúde (EPS) junto aos agentes comunitários de saúde (ACS) em resposta às necessidades de mudança na detecção precoce de demência na fase leve fundamentando-se na aprendizagem significativa e na instituição de ambientes pedagógicos que promovam a problematização das práticas, com o objetivo de avaliar, revisar e transformar os processos de trabalho até então praticados $^{4}$.

O objetivo do estudo foi analisar a oficina de capacitação do ACS para qualificação e identificação de casos de demências na fase leve.

\section{REVISÃO DE LITERATURA}

$O$ cuidado às pessoas com demências é um desafio da atenção básica $(A B)^{5}$. Em todo mundo, a demência tem sido sub-diagnosticada e o diagnóstico geralmente feito em um estágio avançado da doença na clínica especializada. Tal fato tem causas multifacetadas e envolve desde a falta de formação e treinamento dos profissionais da saúde inseridos na $A B$ até a triagem, diagnóstico e tratamento farmacológico/não farmacológico dos novos casos ${ }^{6}$. Mesmo em países desenvolvidos, apenas um quinto dos diagnósticos são rotineiramente reconhecidos e documentados na atenção primária à saúde ${ }^{7}$.

Para que isso passe a ser uma realidade, destaca-se a necessidade de articulação de uma política de saúde, programas e estratégias de saúde que respondam ao amplo e complexo impacto dessa doença na sociedade.

A secretaria municipal da saúde (SMS) da cidade de São Paulo (SP) implementou a área técnica de saúde da pessoa idosa, que tem como uma de suas atribuições a implantação e a implementação de cursos de capacitação na área de envelhecimento e saúde da pessoa idosa, a partir da formação continuada para níveis superior, médio e básico ${ }^{8}$

O ACS como profissional da estratégia de saúde da família (ESF) é um importante elo com a comunidade, pois é quem faz visita ao domicílio, realiza o mapeamento de áreas, cadastra as famílias e promove a troca de saberes entre as pessoas da comunidade e os outros profissionais do serviço de saúde ${ }^{9,10}$. Ele deve ser capacitado sobre os diferentes aspectos do processo saúde - doença, entre eles, o saber biomédico e o conhecimento quanto à interação que se deve ter com as famílias e suas necessidades.

Dessa forma, deve existir um constante movimento no sentido de garantir a esses profissionais a possibilidade de desenvolver plenamente suas potencialidades, estimulando-os a realizar um trabalho comunitário participativo, reflexivo e transformador ${ }^{11}$.

A importância da qualificação e formação desse profissional para a detecção da demência na fase leve pode facilitar o acesso à informação, orientação e encaminhamento, apoio e acolhimento às pessoas com provável demências e suas famílias ${ }^{12}$.

\section{METODOLOGIA}

Aplicou-se o método descritivo com intervenção (oficinas), comparando o desempenho dos sujeitos no pré e pós oficina. Foi realizada uma oficina de capacitação aos ACS no município de Santos na assistência às pessoas com demências e suas famílias. O projeto foi submetido e aprovado pela Coordenadoria de Formação Continuada em Saúde (COFORM) da cidade de Santos e pelo Comitê de Ética em Pesquisa com seres humanos, sob o parecer no 2.577.483.

Em seguida, foi contatada a coordenação da unidade básica de saúde (UBS) do Embaré para fechamento de datas e disponibilidade dos ACS. Os critérios de inclusão foram: ser ACS da UBS Embaré, ter disponibilidade de horário, ter interesse em participar da oficina e concordar com o termo de consentimento livre esclarecido (TCLE). Os critérios de exclusão foram: ausência em dois e/ou mais encontros, não concordância com o TCLE. Participaram da oficina, oito ACS da referida unidade após a explicação dos objetivos e princípios éticos, seguida da assinatura do TCLE. Foram realizados quatro encontros semanais, de cinco horas cada, totalizando 20 horas de capacitação, no período de abril a maio de 2018. Cada encontro abordou aspectos centrais das demências que foram divididos em tópicos de acordo com a proposta de cada encontro.

A coleta de dados se deu por meio de dois questionários (pré e pós-oficina) semiestruturados, com dados sociodemográficos dos participantes, como idade e nível de escolaridade, além de perguntas abertas com informações sobre a atuação dos ACS na unidade básica de saúde (UBS), a demanda de idosos atendidos na região e o nível de compreensão sobre as síndromes demenciais.

Todos os encontros foram gravados e transcritos. Foi utilizada a análise de conteúdo temática com os seguintes passos: pré análise - articulação dos objetivos iniciais com o processo de pesquisa, para potencializar o resultado final; 
exploração do material - delimitação dos conteúdos eminentes e da ordem em que serão abordados; tratamento dos resultados obtidos e interpretação - inter-relação entre resultados colhidos e materiais teóricos previamente selecionados e/ou notado pela análise ${ }^{13}$.

A primeira oficina contou com a participação dos nove ACS da UBS Embaré. Todos foram orientados quanto aos objetivos da oficina e esclarecidos sobre os princípios éticos. Nesse encontro, foram propostas a apresentação dos integrantes e a explicação das atividades que seriam desenvolvidas conforme o cronograma, além das regras para a participação, como por exemplo: o limite de uma falta para o recebimento do certificado de capacitação.

Os participantes foram solicitados a preencher o questionário pré - oficina. Em seguida, foram introduzidas informações centrais referentes às síndromes demenciais, como: definição, apresentação do Diagnostical and Statistical Manual of Mental Disorders (DSM) III/V, prevalência e incidência no cenário brasileiro, fatores de risco e possibilidades de tratamento e diagnóstico/prognóstico. Para isso foi utilizado como dinâmica uma apresentação dialogada por meio de uma roda de conversa. Dessa forma, na medida em que as informações eram transmitidas, todos tinham a liberdade para pontuar e/ou questionar aspectos que traziam dúvidas 14,15.

No segundo encontro, os cinco presentes justificaram a ausência dos demais pela aplicação de escalas durante campanhas para auxiliar na organização de filas e distribuição de senhas.

As temáticas abordadas nesse encontro foram diagnóstico clínico, avaliação, tratamento farmacológico e não farmacológico, prognóstico, prevenção, estágios e classificação dos principais subtipos das demências (demência da Doença de Alzheimer (DA), demência vascular (DV), demência de corpos de lewy (DCL) e demência fronto-temporal $(\mathrm{DFT})^{16}$. Apesar do número reduzido, todos articularam as discussões, a partir de casos de idosos acompanhados no território.

Para o terceiro encontro foi proposta a apresentação do Mini Exame do Estado Mental (MEEM) ${ }^{17}$. Os oito participantes foram divididos em dois grupos, com quatro em cada um com duração de 2 horas e meia para que todos pudessem participar ativamente da oficina.

Inicialmente foram feitas algumas perguntas em relação às temáticas já abordadas, no intuito de conhecer dúvidas sobre as informações até então discutidas.

O último encontro teve a presença de oito ACS que foram divididos em dois subgrupos novamente. Conforme proposto na metodologia, cada um dos ACS respondeu o questionário de avaliação pós-oficina. Ainda como técnica de avaliação da oficina, ao longo dos encontros, foram feitas perguntas disparadoras que esclareceram dúvidas remanescentes e possibilitaram uma melhor compreensão de algumas informações. Foi aberto um espaço para críticas relativas à metodologia da oficina, ao cronograma, horários e números de encontros. No fechamento, foram apresentados os serviços de atenção aos idosos e suas famílias e/ou cuidadores, na região da Baixada Santista.

\section{RESULTADOS E DISCUSSÃO}

Participaram inicialmente nove ACS da UBS do Embaré/ Santos, de acordo com consulta prévia de agenda e horários disponíveis. Um dos participantes não preencheu os critérios de inclusão pois faltou em dois dos encontros, devido às demandas da unidade para ações de campanha preventiva da gripe. Dos oito participantes restantes, sete eram mulheres e um homem.

Em relação à faixa etária, três dos participantes tinham entre 40 a 50 anos, dois deles entre 50 a 60 anos e três acima de 60 anos. Para o tempo de exercício no cargo, os oito participantes tinham em média 16 anos de carreira, porém seis deles com menos de 8 meses na UBS/Embaré. A referida unidade cobre as regiões do Embaré, Macuco e Estuário. Dentre os ACS, cinco atendiam famílias no território do Embaré, dois no Macuco e Estuário e um ainda não tinha região definida.

A partir da análise de conteúdo dos depoimentos relativos às questões abertas, emergiram três categorias temáticas: o papel do ACS na detecção da demência, fase leve; a percepção dos ACS em relação à demência; e possibilidades de articulação do tema na atenção básica. As categorias são tratadas a seguir.

\section{O papel do ACS na detecção da demência, fase leve \\ Pré-oficina}

O Ministério da Saúde (MS) define como função do ACS facilitar o vínculo entre as famílias e o serviço de saúde. Residir no território, torna essa aproximação mais viável ${ }^{18}$. Inicialmente, os relatos dos ACS corresponderam à importância do seu papel no serviço de saúde para a escuta e acolhimento ao munícipe:

\section{[...] é por isso que a UBS coloca a gente na linha de frente. (ACS 2)}

Porém, o fato de estar no mesmo território dos usuários foi apontado como aspecto negativo da profissão, como 
referiu um depoente:

Eu não gosto. Às vezes, a pessoa me encontra no mercado e fala: 'olha o médico marcou essa consulta para mim [...]' não respeitam sábado, domingo nem feriado. (ACS3)

Neste estudo, os agentes referiram ser abordados em horários fora do expediente diante da facilidade que a comunidade tem em acessá-los para tirar dúvidas em relação às consultas e exames. Esses resultados são confirmados em outros estudos $4,9,11$.

Apesar de terem suas funções bem delimitadas pelo MS, estudo realizado em Campinas corroborou tais achados ao ressaltar que na prática, esses profissionais têm suas funções extrapoladas para além daquelas pré-estabelecidas ${ }^{18}$. Na oficina proposta, o cronograma foi alterado em virtude da reorganização dos ACS para a campanha de vacinação da gripe.

Quando questionados sobre o papel dos ACS frente às doenças comuns ao processo de envelhecimento, como as demências, nenhum dos participantes soube identificar qualquer possibilidade de ação diante dessa demanda específica.

O relatório da World Alzheimer Report identifica que o desconhecimento em relação a demência faz parte do estigma público e impacta no atraso do diagnóstico e encaminhamentos aos serviços e tratamentos adequados ${ }^{19}$. Esse estigma também é salientado na literatura sobre demência ${ }^{1,2,5-7,12,20}$.

\section{Pós-oficina}

Os ACS passaram a compreender a importância de seu papel frente às demências a partir da identificação dos sintomas mais comuns, do rastreio cognitivo e das alterações de comportamento explicitados. Sete demonstraram uma melhor compreensão da síndrome demencial, salientando as possibilidades de rastreio e aptidões para o reconhecimento de possíveis casos.

Vai depender do resultado do teste de memória, da observação do comportamento do idoso, do jeito que conversa e do que a família vai dizendo. (ACS 3 )

A aplicação de instrumentos de rastreio de memória e de cognição além de uma entrevista com familiar e/ou acompanhante da pessoa idosa tem sido recomendada, na atenção básica, no intuito de realizar seguimentos dos casos positivos do tipo não demência e encaminhamentos à clínica especializada para aqueles com quadro clínico de demência ${ }^{21}$.

Em relação às possibilidades de contribuição para o diagnóstico na fase leve da demência, dos oito participantes, apenas seis responderam. Destes, quatro acreditaram contribuir apenas com orientação às famílias; um descreveu que a partir da avaliação de rastreio, da observação do comportamento do idoso e conversa com a família; e um outro valorizou a avaliação de rastreio, o encaminhamento para UBS, ambulatório de especialidades e o suporte à família. Todos sugeriram maior carga horária da oficina para um melhor aproveitamento e apropriação dos conteúdos relacionados à doença.

Foi possível observar que, mesmo após as dinâmicas oferecidas, no intuito de instrumentalizá-los, os profissionais ainda não se sentiam seguros para identificação e abordagem dos usuários. Possivelmente, a oferta de uma oficina com a carga horária de 20 horas tenha sido insuficiente para a complexidade das questões envolvidas na demência. Outro fator negativo apontado foi a realização da oficina em horário e local do expediente, visto que eram constantemente acionados a sair da sala.

\section{A percepção dos ACS em relação à demência}

Pré-oficina

A falta de informação e capacitação acerca da doença pode gerar ou reforçar, até mesmo nos profissionais de saúde, falsas crenças inclusive de que a demência é uma doença comum ou normal do processo de envelhecimento, segundo o relato a seguir:

\section{Isso faz parte da natureza do ser humano. 90/80 anos, é normal. (ACS3)}

Vale reforça que "a pessoa idosa com suspeita de demência, após avaliação na UBS, deve ser encaminhada para a atenção especializada, respeitando-se os fluxos de referência" 20: 113. Entretanto, a falta de investimento em educação permanente voltada aos profissionais da atenção básica gera uma busca maior pela assistência especializada e, consequentemente, longas filas de esperas.

O fato de três dos participantes terem idade acima de 60 anos favoreceu a sensibilização e conscientização de que a demência é um problema prioritário de saúde pública diante do envelhecimento populacional. Eis outro depoimento:

[...] e vem aquela pergunta: quem é que vai cuidar da gente se eu ficar assim? (ACS5) 


\title{
Pós-oficina
}

Durante as dinâmicas desenvolvidas, eles identificaram situações de visitas domiciliares de rotina em que poderiam aplicar os testes de rastreio e escuta direcionada à família para possíveis seguimentos.

Acho que tem um munícipe com Alzheimer. Ele só lembra do passado. Se não é ela [a esposa] que tenta dar banho, ele não toma porque diz 'já tomei, já tomei' só que ele não tomou [...] (ACS 6)

Nos encontros, foi identificado que os ACS comumente associavam a demência aos quadros clínicos de outras condições de saúde como por exemplo, o delirium de um quadro infeccioso:

\begin{abstract}
Um dia minha mãe estava falando coisas sem sentido. Eu pensei que ela estava com demência [...] quando vi estava com quase 40 graus de febre. No pronto socorro identificaram por meio de exame de urina que ela estava com infecção. Já estava quase subindo para os rins [...] depois que deram o remédio ela ficou normal. (ACS 6)
\end{abstract}

Quando questionados sobre como identificar um possível caso de demência, apenas seis responderam, sendo que somente um demonstrou consistência em sua resposta, a partir das discussões realizadas.

Ficou evidente a percepção do estigma em relação à demência, a dificuldade de compreensão dos fatores que envolvem um quadro demencial e da percepção da família como elemento fundamental nesse processo.

\section{Possibilidades de articulação do tema na atenção básica}

\section{Pré-oficina}

Em resposta à pergunta Quais os problemas de saúde mais comuns aos idosos que você acompanha? prevaleceram a hipertensão e diabetes, seguidas de artrose/osteoporose, acidente vascular encefálico e, por último, a demência, sendo citada por apenas um deles.

Quanto aos encaminhamentos de casos de demência para a rede de saúde, as respostas apontaram: referenciar ao clínico geral da UBS; encaminhar diretamente ao neurologista; ao Centro de Atenção Psicossocial (CAPS) e apenas um mencionou o enfermeiro chefe, como a possibilidade mais viável para pensar em desdobramentos junto à clínica especializada.

A gente não fala encaixe aqui, mas elas [enfermeiras] têm como pedir para essa pessoa passar no clínico imediatamente. Só que para frente já bloqueia quem vai para o neurologista. (ACS 2)

Pesquisa desenvolvida na Inglaterra sugeriu que investimentos na formação dos profissionais na atenção básica podem minimizar a sobrecarga no atendimento às pessoas com alterações de memórias e de cognição na assistência especializada 22

Pós-oficina

Foi possível identificar a conscientização dos ACS perante as necessidades de articulação desse problema de saúde pública na atenção básica. Dois deles relataram ser fundamental o investimento em oficinas de capacitação; dois sugeriram ações de educativas às famílias; um considerou importante a implementação de programa de educação permanente sobre a deteç̧ão da demência na atenção básica. Estudo identificou outras práticas inovadoras que vão ao encontro com as comunidades amigas da pessoa com demência, como a realização de eventos para informar e conscientizar a população, e a criação de clínicas de memórias, nas quais qualquer um dos profissionais da equipe pode ser instrumentalizado para aplicar instrumentos de rastreio cognitivo ${ }^{22}$.

O crescente envelhecimento populacional mundial e sua relação direta com doenças crônico-degenerativas como as demências, têm despertado campanhas em todo mundo pela Organização Mundial de Saúde e Alzheimer Disease International (ADI), no sentido de sensibilizar gestores para a criação de planos globais de ação que possam culminar com a criação de políticas públicas sobre a prevenção e tratamento precoce das demências ou que esta seja contemplada em outras políticas já implementadas ${ }^{23}$.

\section{CONCLUSÃO}

Os profissionais ACS são um importante elo entre a equipe básica de saúde e a população em geral. O elevado número de idosos na cidade de Santos traz estimativas de taxas elevadas de incidência e prevalência das demências. É primordial a capacitação dos agentes para a detecção das doenças crônicas degenerativas a partir da adoção da educação permanente como estratégia fundamental à recomposição das práticas de formação, de atenção e de formulação de políticas públicas.

A oficina de capacitação mostrou-se recurso potente na formação dos ACS para rastreio e identificação das pessoas com demências na atenção básica. 


\section{REFERÊNCIAS}

1. Custódio N, Wheelock A, Thumala D, Slachevsky A. Dementia in Latin America: epidemiological evidence and implications for public policy. Frontiers Aging Neuroscience. 2017 [cited 2018 Dec 05]; (13): 9-221. Available from: https://www.ncbi.nlm.nih.gov/pmc/articles/PMC5508025/

2. Organização Mundial de Saúde. Dementia: a public health priority. World health organization and alzheimer's disease international. 2012 [cited 2018 Sep 10]. Available from: https://apps.who.int/iris/bitstream/handle/10665/75263/9789241564458 eng.pdf?sequence $=1$

3. Prefeitura de Santos. 2017 [cited 2019 Sep 29]. Available from: https://www.santos.sp.gov.br/?q=noticia/santos-e-a-melhorpara-idoso-viver-e-exemplo-para-outras-cidades

4. Fagundes NC, Rangel AGC, Carneiro TM, Castro LMC, Gomes BS. Continuing professional development in health for working nurses. Rev. enferm. UERJ. 2016 [cited 2019 Sep 27]; 24(1):e11349. DOI: http://dx.doi.org/10.12957/reueri.2016.11349

5. Poon NY, Ooi CH, How CH, Yoon PS. Dementia management: a brief overview for primary care clinicians. Singapore Med. J. 2018 [cited 2019 Set 27]; 59(6): 295-9. DOI: https://doi.org/10.11622/smedi.2018070

6. Drummond N, Birtwhistle R, Williamson T, Khan S, Garies S, Molnar F. Prevalence and management of dementia in primary care practices with electronic medical records: a report from the canadian primary care sentinel surveillance network. Camaj. OPEN. 2016 [cited 2019 Sep 27]; 4(2). DOI: https://doi.org/10.9778/cmaio.20150050

7. Miranda GMD, Mendes ACG, Silva ALA. Population aging in Brazil: current and future social challenges and consequences. Rev. Bras. de Geriatria e Gerontologia. 2016 [cited 2018 Sep 10]; 19(3):507-19. Available from: http://www.scielo.br/pdf/rbgg/v19n3/pt 1809-9823-rbgg-19-03-00507.pdf

8. Secretaria de Saúde do Estado de São Paulo. Documento norteador: unidade de referência à saúde do idoso do município de São Paulo. [Internet]. Updated 2016 Dez 20. São Paulo. [cited 2018 Sep 10] Available from: https://www.prefeitura.sp.gov.br/cidade/secretarias/saude/atencao basica/pessoa idosa/index.php?p=5432

9. Placideli N, Ruiz T. Continuing Education in gerontology for community health agent. Rev. Bras. Med. Família e Comunidade. 2015 [cited 2018 Sep 10]; 10(36): 1-10. DOI: http://dx.doi.org/10.5712/rbmfc10(36)948

10. Ministério da Saúde (Br). Secretaria de Assistência à Saúde. Saúde da família: uma estratégia para reorientação do modelo assistencial. Brasília, 1997 [cited 2018 Sep 10]. Available from: https://pt.slideshare.net/institutoconscienciago/sade-da-famliauma-estratgia-para-a-reorientao-do-modelo-assistencial

11. Fortes KMGS, Moura MEB, Nunes BMVT, Landim CAP, Lago EC. Training of the community family health agent in elderly assistance. Rev. enferm. UFPE [Internet], 2016[cited 2019 Sep 27]; 10(1): 211-217. Available from: https://periodicos.ufpe.br/revistas/revistaenfermagem/article/viewFile/10942/12246

12. Pessoa RMP, Faria SM, Morais D, Chagas MHN. From dementia to major neurocognitive disorder: current aspects. Rev. Ciênc. saúde. 2016 [cited 2018 Sep 10]; 6(4). Available from: http://186.225.220.186:8484/index.php/rcsfmit zero/article/download/606/384

13. Minayo MCS. O desafio do conhecimento: pesquisa qualitativa em saúde. São Paulo: HUCITEC; 2014. p. 303-18.

14. American Psychiatric Association (APA). Diagnostic and statistical manual of mental disorders. $3^{\text {rd }}$ ed. Washington (DC): American Psychiatric Press; 1980.

15. American Psychiatric Association (APA). Manual diagnóstico e estatístico de transtornos mentais: DSM-5. 5.ed. Porto Alegre (RS): Artmed; 2014.

16. Parmera JB. Nitrini R. Investigation and diagnostic evaluation of a patient with dementia. Rev. Med. 2015 [cited 2018 Sep 10]; 94(3):179-84. DOI: https://doi.org/10.11606/issn.1679-9836.v94i3p179-184

17. Brucki SMD, Nitrini R, Caramelli P, Bertolucci PHF, Okamoto IH. Suggestions for utilization of the mini-mental state examination in Brazil. Arq. Neuro-Psiquiatr. [Internet]. 2003 [cited 2018 Set 10], 6(3):777-81. DOI: http://dx.doi.org/10.1590/S0004$282 \times 2003000500014$.

18. Nascimento EPL, Correa CBS. Community health agents: training, involvement, and practices. Cad. Saúde Pública. 2008 [cited 2018 Sep 10]; 24(6):1304-13. Available from: http://bvsms.saude.gov.br/bvs/is digital/is 0308/pdfs/IS28(3)078.pdf

19. Alzheimer Disease International (ADI). Atittudes to dementia. London (UK): World Alzheimer Report; 2019 [cited 2019 Sep 26 ]. Available from: https://www.alz.co.uk/research/WorldAlzheimerReport2019.pdf

20. Ministério da Saúde (Br). Secretaria de Assistência à Saúde. Departamento de atenção básica. Envelhecimento e saúde da pessoa idosa. Brasília (DF): Editora MS; 2006 [cited 2018 Sep 10]; 1:113. Available from: http://bvsms.saude.gov.br/bvs/publicacoes/evelhecimento saude pessoa idosa.pdf

21. Grober E, Wakefield D, Ehrlichc AR, Mabie P, Lipton RB. Identifying memory impairment and early dementia in primary care. Alzheimer's \& Dementia: Diagnosis, Assessment \& Disease Monitoring. 2017[cited 2019 Sep 27]; 6:188-95. DOI: http://dx.doi.org/10.1016/i.dadm.2017.01.006

22. Wells CE, Smith SJ. Diagnostic care pathways in dementia: a review of the involvement of primary care in practice and innovation. Journal of Primary Care \& Community Health. 2017[cited 2019 Sep 27]; 8(2) 103-11. DOI: https://doi.org/10.1177/2150131916678715

23. Alzheimer Disease International (ADI). From plan to impact. Progress towards targets of the global action plan on dementia. London (UK): ADI; 2018 [cited 2018 Sep 10]. Available from: https://www.alz.co.uk/adi/pdf/from-plan-to-impact-2018.pdf 